\title{
Efficacy and Safety of Fusion Imaging in Radiofrequency Ablation of Hepatocellular Carcinoma Compared to Ultrasound: A Meta-Analysis
}

\author{
Tao Jie ${ }^{1 \dagger}$, Feng Guoying ${ }^{1 \dagger}$, Tang Gang ${ }^{1}$, Shi Zhengrong ${ }^{2 *}$ and Li Maoping ${ }^{3 *}$ \\ ${ }^{1}$ Department of General Surgery, Chongqing Medical University, Chongqing, China, ${ }^{2}$ Department of General Surgery, The \\ First Affiliated Hospital of Chongqing Medical University, Chongqing, China, ${ }^{3}$ Department of Ultrasound, The First Affiliated \\ Hospital of Chongqing Medical University, Chongqing, China
}

\section{OPEN ACCESS}

Edited by:

Patrick J. Schuler,

Ulm University Medical

Center, Germany

Reviewed by:

Xiao-Wan Bo,

Tongji University, China

Thanongchai Siriapisith,

Mahidol University, Thailand

*Correspondence:

Li Maoping

limaoping@hospital.cqmu.edu.cn

Shi Zhengrong

shizr@hospital.cqmu.edu.cn

tThese authors have contributed equally to this work and share first authorship

Specialty section:

This article was submitted to Surgical Oncology,

a section of the journal Frontiers in Surgery

Received: 20 June 2021 Accepted: 18 October 2021 Published: 06 December 2021

Citation:

Jie T, Guoying F, Gang T, Zhengrong S and Maoping L (2021) Efficacy and Safety of Fusion Imaging in

Radiofrequency Ablation of

Hepatocellular Carcinoma Compared to Ultrasound: A Meta-Analysis. Front. Surg. 8:728098. doi: 10.3389/fsurg.2021.728098
Background: Radiofrequency ablation (RFA), generally performed under real-time guidance of ultrasound which is safe and effective, is a common minimally invasive therapy for treating hepatocellular carcinoma. Fusion imaging (FI) is a newly developed imaging method, which integrates CT/MRI accurate imaging and matches the characteristics of real-time ultrasound imaging, thereby providing a new approach to guide tumor ablation therapy. However, the efficacy and safety of Fl as opposed to ultrasound in tumor ablation remains unclear.

Objective: The present study sought to evaluate the difference in the efficacy and safety between $\mathrm{Fl}$ and ultrasound in radiofrequency surgery for the treatment of hepatocellular carcinoma through a metaanalysis.

Materials and Methods: Searching for studies comparing the efficacy and safety of $\mathrm{FI}$ and ultrasound in radiofrequency of hepatocellular carcinoma in PubMed, Embase, and Cochrane Library databases for articles published until April 2021. Random or fixed effect models were used for statistical analysis. Metaanalysis and sensitivity analysis were used on the included studies.

Results: A total of six studies met predefined inclusion criteria, and were finally included in the analysis. Sensitivity and subgroup analyses, based on predetermined patient characteristics, allowed minimization of bias. In the RFA of hepatocellular carcinoma, FI decreased 1-year overall survival (OS) when compared with ultrasound. But FI was not significantly different from ultrasound in terms of technical efficiency, 1-, 2-, and 3-year local tumor progression (LTP), complications, as well as 2-year OS. Subgroup analysis, based on tumor mean diameter, showed that $\mathrm{Fl}$ reduced the rate of 1 - and 2-year LTP in patients with tumors of mean diameter $\geq 15 \mathrm{~mm}$ when compared with ultrasound. Moreover, operative complications could be reduced in patients with tumor mean diameter $<15 \mathrm{~mm}$ using $\mathrm{Fl}$, compared with ultrasound.

Conclusion: Overall, these results showed that FI may have some effects on improving efficacy and safety of thermal ablation in HCC patients, relative to ultrasound. However, it may be a more effective method for managing large lesions, as well as those that are difficult to ablate. Further large-scale and well-designed randomized controlled trials are needed to validate these findings.

Keywords: curative effect, fusion imaging, meta-analysis, security, ultrasound 


\section{INTRODUCTION}

Radiofrequency ablation (RFA) is a safe and effective method for treating patients with early hepatocellular carcinoma who cannot tolerate surgery or are reluctant to undergo surgery $(1,2)$, while imaging holds the key to the curative effect and prognosis of frequency ablation (3). On the other hand, ultrasound (US) remains the most commonly used imaging technique $(4,5)$ due to its economic convenience, nonionizing radiation, and realtime characteristics $(6,7)$. However, the imaging of ultrasound is relatively fuzzy in the face of lesions $<2 \mathrm{~cm}$ (8), isoechoic, located in the center or top of the liver, as well as interference from adjacent structures and tissues (9). Therefore, contrast-enhanced ultrasound (CEUS) is required. However, the positioning ability for CEUS is also limited for tumors with poor blood supply and the situation where it is difficult to evaluate the ablation range, which may lead to tumor residue (10). In addition, tumors that are not visible in ultrasound, remain a major challenge during RFA (8). Therefore, RFA under ultrasound alone is constrained by numerous limitations.

Advancements in computer graphics, 3D image processing technology, and the emergence of fusion imaging (FI) have all improved RFA (11). FI, which overlaps images from different image sources and combines real-time images of ultrasound with the high resolution of CT/MRI (9), has been developed. Notably, this technique is more accurate than ultrasound alone in identifying target lesions, thereby allowing ablation of that are invisible or ablate tumors that are difficult to ablate (8). In addition, FI can also determine the ablation edge and evaluate treatment response in real time (12), has excellent efficacy and safety $(13,14)$, and may become an important imaging technique for RFA of hepatocellular carcinoma. In fact, the technique can also be used in needle biopsy for disease diagnosis (15), and is also a promising application in prostate (16), liver (17), heart (18), and brain diseases (19), among others.

However, published studies have yielded conflicting results with regards to efficacy and safety of FI relative to that of ultrasound. While some studies have shown that FI is more superior than ultrasound $(20,21)$, others have found no significant differences in the two technologies $(22,23)$. Therefore, the present metaanalysis was designed to systematically evaluate efficacy and safety of FI relative to ultrasound in radiofrequency surgery for the treatment of hepatocellular carcinoma. Specifically, patients with hepatocellular carcinoma undergoing RFA were selected as the research objects, and technical efficiency, local tumor progression (LTP), and complications [thoracic hemorrhage, biliary injury, so on (24)] were taken as the main evaluation indexes, whereas survival (OS) was considered the secondary evaluation.

\section{MATERIALS AND METHODS}

\section{Literature Search}

Articles from PubMed, Embase, and Cochrane library databases were searched and relevant articles were retrieved. Search strategy involved the following keywords: hepatocellular carcinoma, RFA, FI, and ultrasound. Due to the relatively new development of
FI in hepatocellular carcinoma radiofrequency, studies published were screened until April 1, 2021, and studies published after that date were not included. References of selected literatures were also screened to prevent the omission of relevant studies. In the initial screening, we read the title and abstract to determine if it met our inclusion criteria. The available full-text articles were then reviewed as described (Figure 1). Specifically, two reviewers (Tao and Tang) conducted literature retrieval and data extraction, and any questions were resolved through discussion with other reviewers (Feng and Shi and $\mathrm{Li}$ ).

\section{Inclusion and Exclusion Criteria}

Inclusion criteria were: (1) Studies to compare application of FI and ultrasound or FI and CEUS in RFA of patients with nonrecurrent hepatocellular carcinoma; (2) reported results included at least one of the technical efficiencies, LTP, complications, and OS. Exclusion criteria were: (1) nonEnglish papers or repetitive articles; (2) Unpublished data or gray literature including conference abstracts, dissertation, brief reports, book chapters, editorials, and patents. Any discrepancies regarding selection of a qualified article were resolved through discussion or consultation with other reviewers (Feng and shi and li).

\section{Data Extraction}

To reduce chances of human error, data extraction for each study was performed independently by two reviewers (Tao and Tang) using a developed form. Data collected included, name of the first author, study design, country, and year of publication, sample diameter, participants' gender, and their mean age, liver function (Child-Pugh class), tumor diameter, type of intervention in the control group, and main outcomes. There were minimal disagreements between the two researchers with regards to data extraction or quality assessment, and these issues were resolved through discussion and consensus.

\section{Quality Assessment}

Since the included articles included both a cohort study and a randomized controlled studies, the two reviewers (Tao and Tang) evaluated the quality of each cohort study using the Newcastle-Ottawa quality assessment scale, whereas that of randomized controlled studies was performed using the risk of bias assessment scale in the Cochrane manual of systematic evaluation of interventions. If both reviewers had different views on the results, following the separate assessment, other researchers (Feng and Shi and $\mathrm{Li}$ ) were called upon to help to reach a consensus. Quality of the cohort studies was assessed based on three factors, namely selection, comparability and outcome. On the other hand, risk of bias in randomized controlled studies was assessed based on seven criteria, namely randomization, distribution hiding, blindness of participants and operators, detection blindness, incomplete data, selective reporting, and other biases.

\section{Statistical Analyses}

The primary endpoints in the meta-analysis included technical efficiency, 1-, 2-, and 3-year LTP, and complications, whereas 

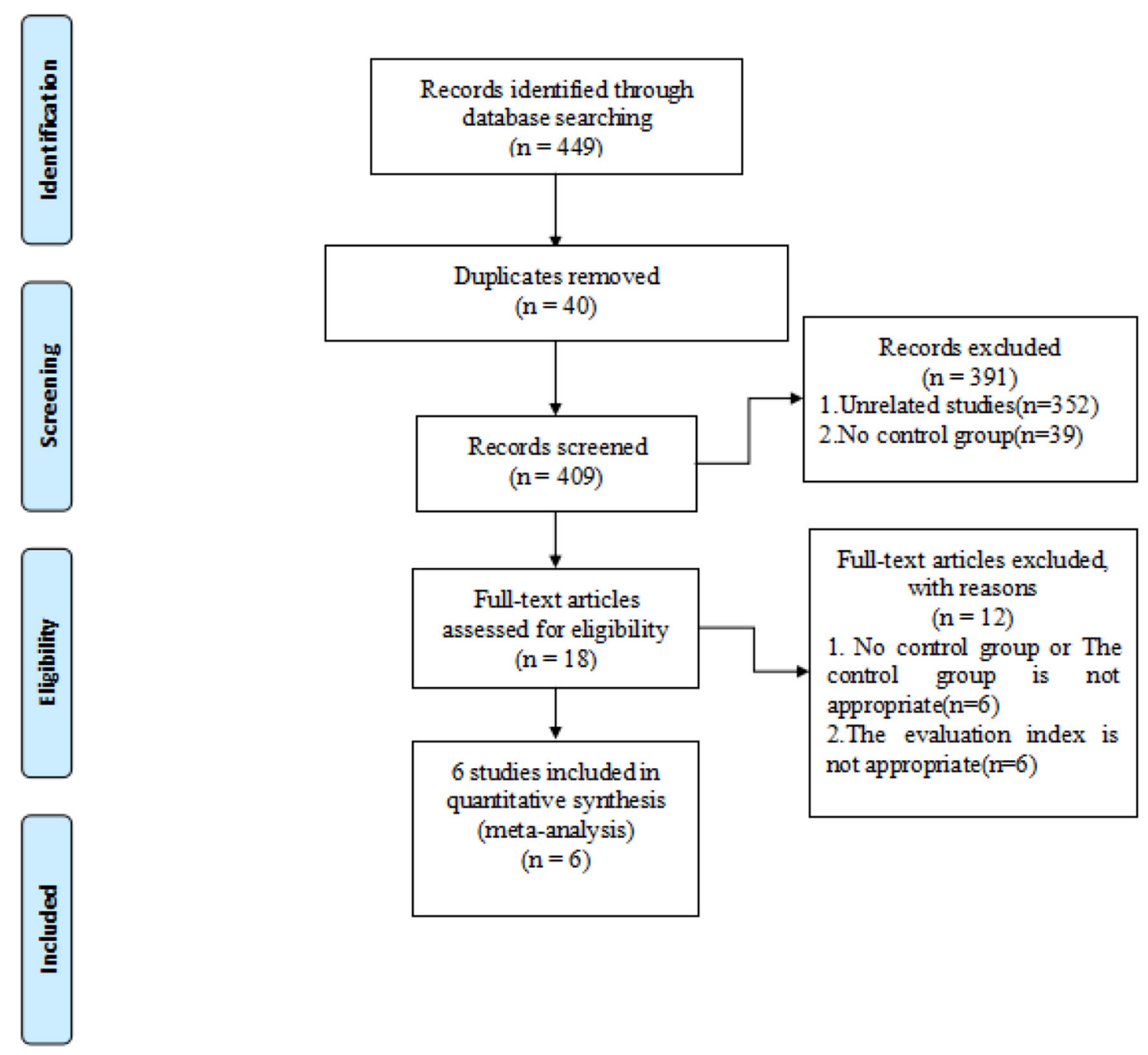

FIGURE 1 | PRISMA flow diagram of study selection process.

secondary endpoints were 1- and 2-year OS. Heterogeneity among studies was assessed using the $I$-square $\left(I^{2}\right)$ and $Q$ tests. When $I^{2}<50 \%$ and $P>0.1$ in the $Q$-value test, a fixed-effect model was applied. Otherwise, the random effects model was employed. Assessment of potential sources of interstudy heterogeneity was performed using subgroup analyses, based on baseline tumor mean diameter (tumor mean diameters $\geq 15$ and $<15 \mathrm{~mm}$, the data was obtained by looking at all the literatures) and control type (US and CEUS). The proportion of each study to the overall outcome was assessed using sensitivity analysis, while publication bias was evaluated by grade correlation based on Begg and regression asymmetry test of Egger. Statistical analyses were performed using Stata 15.1 software (Statacorp, College Station, Texas, USA) and Review Manager5.3, by two investigators (Tao and Tang), and reviewed by the other researchers (Feng and Shi and $\mathrm{Li}$ ).

\section{RESULTS}

\section{Literature Search}

The selection process of the present study is shown using the PRISMA flow diagram (Figure 1). Search strategy resulted in a total of 449 records, of which 40 were duplicates and were subsequently eliminated. The remaining 409 records were screened by title/abstract, and 18 selected for full-text assessment. An additional 12 records did not meet our inclusion criteria, leaving a final six full-text articles for metaanalysis (10, 21-23, $25,26)$.

\section{Characteristics of the Included Studies}

Characteristics of the included studies, comprising six articles with 1,158 patients aged between 29 and 88 years from Asia, are summarized in Table 1 . The studies were three arm tests $(22,26)$, treating them as four two-arm tests [Huang (22) (1): contrasting 


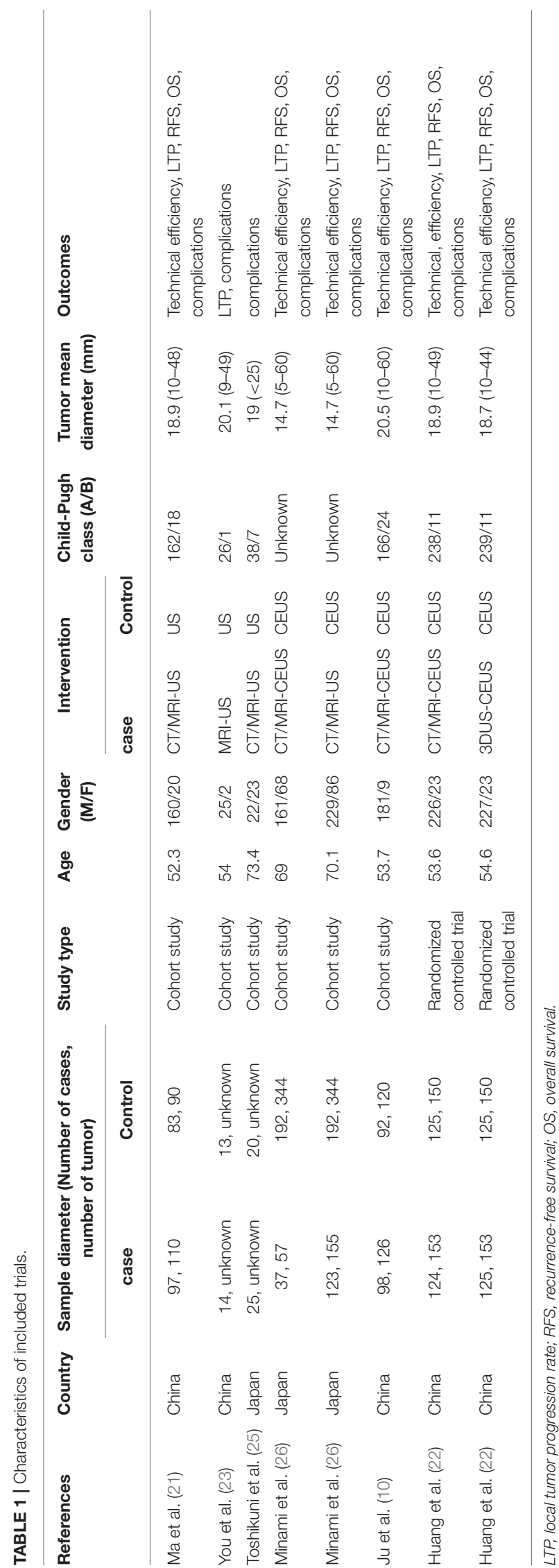

FI combined with CEUS and CEUS; Huang (22) (2): contrasting FI and CEUS; (26) (1): contrasting FI combined with CEUS and CEUS; (26) (2): contrasting FI and CEUS]. Six studies reported 1- and 2-year LTP, four reported on 3-year LTP, while eight analyzed complications of RFA. Moreover, six and two studies were cohort and randomized controlled studies, respectively. Five cohort studies were assessed using the Newcastle-Ottawa quality assessment scale (Table 2), whereas one randomized controlled study was assessed using the Cochrane risk bias assessment tool (Table 3).

\section{Effect on Technical Efficiency}

Pooled effect diameter analysis did not reveal any significant differences in the technical efficiency between ultrasonic image fusion and the control group, across the six included trials (RR, RE: 1.02 ; 95\% CI: 0.98, 1.06, $p=0.28$; Figure 2). However, there was significant heterogeneity between the effect diameter of included studies $\left(I^{2}=83 \%, p<0.0001\right)$. Moreover, subgroup analysis, based on mean diameter $(<15 \mathrm{~mm})$ revealed no heterogeneity $\left(I^{2}=0.0 \%, p=0.77\right)$. Results of sensitivity analysis, used to examine the effect of each study on pooled effect diameter, revealed that exclusion of Ma's study (21) from the analysis altered the overall effect diameter (RR, RE:1; 95\% CI: 0.99, 1.02, $p$ $=0.84)$.

Moreover, no evidence of significant publication bias was found across the included studies with regards to technical efficiency ( $p=0.81$, Begg's test and $p=0.65$, Egger's test). Results of subgroup analyses on technical efficiency are presented in Table 4.

\section{Effect on LTP}

Six trials reported data on 1-year LTP, and their pooled effect diameter based on ultrasonic image fusion, relative to the control group was (OR, RE: 0.67 ; 95\% CI: $0.36,1.25, p=0.21$ ), with a heterogeneity $\left(I^{2}=55 \%, p=0.05\right.$; Figure $\left.3 \mathrm{~A}\right)$. When the metaanalysis was subgrouped by mean diameter, heterogeneity was attenuated in studies with $\geq 15 \mathrm{~mm}\left(I^{2}=42 \%, p=0.16\right.$, test for overall effect: $z=2.1, p=0.04$ ) and in studies with $<15 \mathrm{~mm}$ $\left(I^{2}=0 \%, p=0.67\right)$. Notably, we found significant differences between subgroup heterogeneity $\left(I^{2}=77.8 \%, p=0.03\right)$. Results from sensitivity analysis revealed that exclusion of Ma's study (21) altered the overall effect diameter (OR, RE: 0.86; 95\% CI: 0.52, $1.41, p=0.55)$.

The pooled mean difference for the six datasets, with regards to the effect of ultrasonic image fusion on 2-year LTP, was (OR, RE: $0.61 ; 95 \%$ CI: $0.35,1.07, p=0.08$ ) relative to ultrasonoscopy (Figure 3B), with a heterogeneity of $\left(I^{2}=60.0 \%, p=0.03\right)$. When the metaanalysis was subgrouped by mean diameter, heterogeneity was attenuated in studies with $\geq 15 \mathrm{~mm}\left(I^{2}=40 \%\right.$, $p=0.17$, test for overall effect: $z=2.99, p=0.003$ ), and in studies with $<15 \mathrm{~mm}\left(I^{2}=0 \%, p=0.67\right)$. Then, there was a significant between-subgroup heterogeneity $\left(I^{2}=84.3 \%, p=0.01\right)$. To examine the effect of each study on pooled effect diameter, we performed sensitivity analyses and found that (26) (2)'s study (26) altered the overall effect diameter (OR, RE: 0.50; 95\% CI: 0.31, $0.82, p=0.007)$. 
TABLE 2 | Outcome of assessment of the quality of nonrandomised studies using the Newcastle-Ottawa scale study.

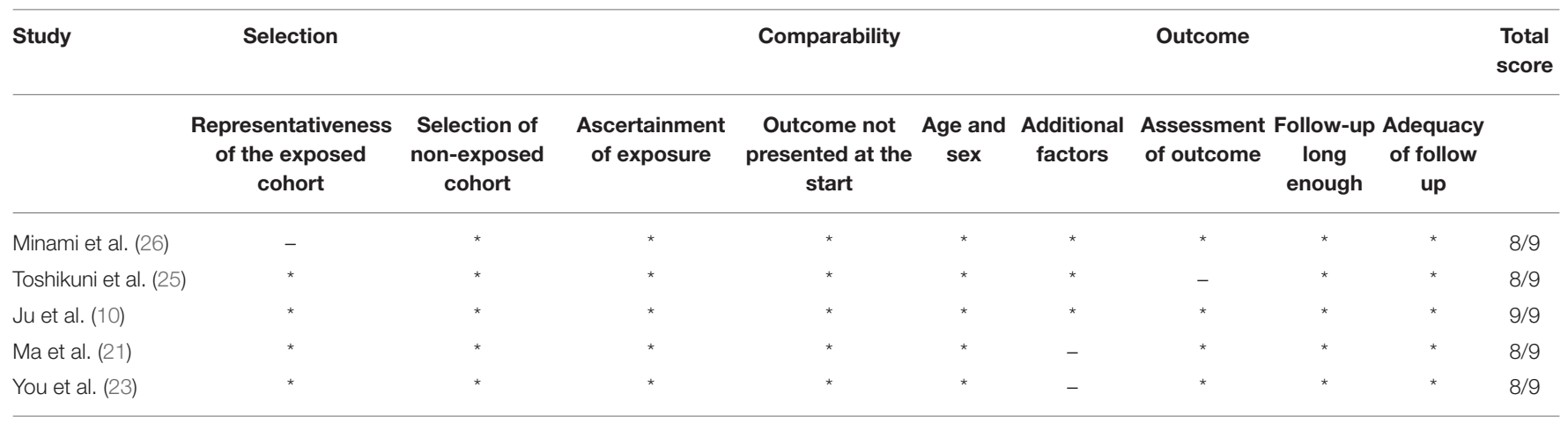

A single asterisk (*) indicates 1 score, and dash (-) indicates 0 score.

TABLE 3 | Risk of bias table.

\begin{tabular}{|c|c|c|}
\hline Bias & Authors' judgement & Support for judgement \\
\hline Random sequence generation (selection bias) & Low risk & Random number \\
\hline Allocation concealment (selection bias) & High risk & Doctors and data collectors know the results of the assignment \\
\hline Blinding of participants and personnel (performance bias) & High risk & No blinded \\
\hline Blinding of outcome assessment (detection bias) & Unclear risk & Insufficient information to judge \\
\hline Incomplete outcome data (attrition bias) & Low risk & Data is balanced between groups \\
\hline Selective reporting (reporting bias) & Low risk & Non-selective reporting \\
\hline Other bias & Low risk & There was no obvious other bias \\
\hline
\end{tabular}

\begin{tabular}{|c|c|c|c|c|c|c|c|c|c|}
\hline Studv or Subgroup & \multicolumn{2}{|c|}{ Experimental } & \multicolumn{2}{|c|}{ Control } & Weight & $\begin{array}{c}\text { Risk Ratio } \\
\text { M-H, Random, 95\% Cl }\end{array}$ & \multicolumn{3}{|c|}{$\begin{array}{c}\text { Risk Ratio } \\
\text { M-H, Random, } 95 \% \mathrm{Cl}\end{array}$} \\
\hline huang $2019(1)$ & 144 & 145 & 144 & 144 & $24.6 \%$ & $0.99[0.97,1.01]$ & $\rightarrow$ & & \\
\hline huang $2019(2)$ & 144 & 144 & 144 & 144 & $25.5 \%$ & $1.00[0.99,1.01]$ & & & \\
\hline јu 2019 & 125 & 126 & 113 & 120 & $18.3 \%$ & $1.05[1.00,1.10]$ & & & \\
\hline $\operatorname{ma} 2019$ & 110 & 110 & 78 & 90 & $11.2 \%$ & $1.15[1.06,1.25]$ & & & \\
\hline minami 2014(1) & 33 & 37 & 177 & 192 & $6.9 \%$ & $0.97[0.86,1.09]$ & & & \\
\hline minami $2014(2)$ & 112 & 123 & 177 & 192 & $13.6 \%$ & $0.99[0.92,1.06]$ & & & \\
\hline Total $(95 \% \mathrm{Cl})$ & & 685 & & 882 & $100.0 \%$ & $1.02[0.98,1.06]$ & & & \\
\hline Total events & 668 & & 833 & & & & & & \\
\hline $\begin{array}{l}\text { Heterogeneity: Tau } \\
\text { Test for overall effec }\end{array}$ & $\begin{array}{l}0.00 ; \text { Chi } \\
Z=1.07\end{array}$ & $\begin{array}{l}=29.79 \\
=0.28\end{array}$ & $d f=5($ & $P<0.0$ & $001) ; I^{2}=$ & $83 \%$ & $\begin{array}{lll}0.85 & 0.9 & 1 \\
\text { Favours [experimental] }\end{array}$ & $\begin{array}{c}1.1 \\
\text { Favours [control] }\end{array}$ & 1.2 \\
\hline
\end{tabular}

We also examined the effect of ultrasonic image fusion on 3year LTP in four clinical trials. Overall, metaanalysis revealed no significant effects of the ultrasonic image fusion on 3-year LTP, relative to the control group (OR, RE: 0.71 ; 95\% CI: $0.29,1.79, p$ $=0.47)$, with heterogeneity across studies $\left(I^{2}=74 \%, p=0.008\right.$; Figure 3C). Moreover, sensitivity analysis showed that excluding Ma's study (21) from the analysis changed the overall effect (OR, RE: $1.14 ; 95 \%$ CI: 0.67, 1.93, $p=0.63$ ).

Begg's and Egger's tests did not reveal evidence of publication bias for LTP across 1 -year ( $p=0.26$ and $p=0.272$, respectively), 2-year ( $p=1.00$ and $p=0.915$, respectively), and 3-year $(p=$ 0.73 and $p=0.901$, respectively) periods. The result of subgroup analysis on LTP are presented in Table 4.

\section{Effect on Complications}

Eight trials reported the effect of ultrasonic image fusion on complications. Metaanalysis showed that ultrasonic image fusion had no significant decrease on the complications (RD, RE: -0.02 ; $95 \%$ CI: $-0.04,0.01, p=0.3$; Figure 4), with a heterogeneity of $\left(I^{2}=67 \%, p=0.004\right)$. When the metaanalysis was subgrouped by mean diameter, heterogeneity was attenuated in studies with $\geq 15 \mathrm{~mm}\left(I^{2}=33 \%, p=0.19\right)$ and studies with $<15 \mathrm{~mm}\left(I^{2}=\right.$ $0 \%, p=0.37$, test for overall effect: $z=2.29, p=0.02)$. Then, there was a significant between-subgroup heterogeneity $\left(I^{2}=\right.$ $77.7 \%, p=0.03)$. In addition, sensitivity analysis revealed that the study by You et al. (23) had a significant influence on the effect value (RD, RE: -0.01 ; 95\% CI: $-0.03,0.01, p=0.45)$. 
TABLE 4 | Subgroup analysis to assess the effects of ultrasonic image fusion on radiofrequency ablation.

\begin{tabular}{|c|c|c|c|c|c|c|}
\hline Indicators & Subgrouped by & The number of studies & Effect diameter & $95 \% \mathrm{Cl}$ & $I^{2}(\%)$ & $\begin{array}{c}P \text { for between subgroup } \\
\text { heterogeneity }\end{array}$ \\
\hline \multirow[t]{3}{*}{ Technical efficiency } & Baseline mean diameter & & & & 39.3 & 0.2 \\
\hline & $\geq 15 \mathrm{~mm}$ & 4 & 1.04 & $0.98,1.1$ & 95 & $<0.0001$ \\
\hline & $<15 \mathrm{~mm}$ & 2 & 0.98 & $0.93,1.04$ & 0 & 0.77 \\
\hline \multirow[t]{3}{*}{ 1-year LTP } & Baseline mean diameter & & & & 77.8 & 0.03 \\
\hline & $\geq 15 \mathrm{~mm}$ & 4 & 0.48 & $0.24,0.95$ & 42 & 0.16 \\
\hline & $<15 \mathrm{~mm}$ & 2 & 1.34 & $0.69,2.62$ & 0 & 0.67 \\
\hline \multirow[t]{3}{*}{ 2-year LTP } & Baseline mean diameter & & & & 84.3 & 0.01 \\
\hline & $\geq 15 \mathrm{~mm}$ & 4 & 0.45 & $0.27,0.76$ & 40 & 0.17 \\
\hline & $<15 \mathrm{~mm}$ & 2 & 1.34 & $0.69,2.62$ & 0 & 0.67 \\
\hline \multirow[t]{6}{*}{ Complications } & Baseline mean diameter & & & & 77.7 & 0.03 \\
\hline & $\geq 15 \mathrm{~mm}$ & 6 & 0 & $-0.02,0.02$ & 33 & 0.19 \\
\hline & $<15 \mathrm{~mm}$ & 2 & -0.07 & $-0.12,-0.01$ & 0 & 0.37 \\
\hline & Control group & & & & 0 & 0.74 \\
\hline & US & 3 & -0.04 & $-0.15,0.07$ & 76 & 0.01 \\
\hline & CEUS & 5 & -0.02 & $-0.05,0.01$ & 68 & 0.01 \\
\hline
\end{tabular}

Notably, there was no publication bias, possibly due to the small sample diameter and short follow-up times reported in the studies. Results of subgroup analyses on complications are presented in Table 4.

\section{Effect on Overall Survival}

Quantitative analysis of overall survival, across four trials, revealed significantly lower 1-year overall survival in the ultrasonic image fusion, relative to the control group (OR, FE: $0.47 ; 95 \%$ CI: $0.23,0.97, p=0.04)$, with no evidence of heterogeneity across the studies $\left(I^{2}=0 \%, p=0.67\right.$; Figure 5A). Moreover, ultrasonic image fusion had no effect on 2-year overall survival of patients across four studies that evaluated this technique, relative to controls (OR, FE: 0.95; 95\% CI: 0.55, 1.63, $p=0.85$; Figure 5B). Low heterogeneity across studies was seen $\left(I^{2}=43 \%, p=0.15\right)$.

Begg's and Egger's tests for 2-year overall survival was ( $p=$ $0.174, p=0.041$, respectively). Due to the small sample diameter and short follow-up time of the studies, publication bias could not be confirmed. There was no evidence of publication bias for 1 -year OS ( $p=0.734$, Begg's test and $p=0.453$, Egger's test). The results of subgroup analysis on overall survival are presented in Table 4.

\section{DISCUSSION}

Hepatocellular carcinoma is now the sixth most common type of cancer, and the fourth most common cause of cancer-related deaths worldwide (27), while early hepatocellular carcinoma and OS with RFA are comparable to surgical resection (28). Moreover, FI, which can apply information obtained from different imaging methods to generate excellent efficacy and safety by combining the advantages of real-time ultrasound and high resolution CT/MRI, may be more useful than ultrasound in RFA (29). Therefore, the metaanalysis systematically analyzed six studies (10, 21-23, 25, 26), comprising 1,168 patients, and found that in the RFA of hepatocellular carcinoma, FI decreased 1-year OS, and thereis no significant changes in the efficiency of ablation technology, 1-3 year LTP, 2-year OS, and complications compared to ultrasound. Notably, there was clinical heterogeneity which might affect the result due to the difference in the type of control group included in the study. Therefore, the article further compared the differences about efficacy and safety of CEUS, FI $(10,22,26)$, and the differences about efficacy and safety of ultrasound and FI in RFA $(21,23,25)$. When studying the efficacy and safety of FI and CEUS, there was also no significant change in the results (technical efficiency, $P=0.84$; 1-year LTP, $P=0.55$; 2-year LTP, $P=0.21$; 3-year LTP, $P=0.63$; Complications, $P=0.3$; 1 -year OS, $P=0.04$; 2 -years $\mathrm{OS}, P=0.18$ ). Since there were few studies comparing FI and ultrasound, only analyzing the complications found no significant difference in the study results $(P=0.52)$.

The high echo of the gas generated by heating immediately after ablation will greatly blur the ultrasound image of the lesion and make the next puncture difficult, while there is no the interference of vaporization in FI. In addition, when the lesion is not obvious in ultrasound examination, FI can clearly show the lesion, which helps to reduce the difficulty of surgery (30, 31). Moreover, FI has been shown to increase visibility of liver lesions, which significantly increases the confidence of operators when performing RFA $(32,33)$, thereby improving surgical outcomes and reducing the associated risks and complications (34). Results of our subgroup analyses corroborated these findings, as evidenced by fewer complications in studies that used FI protocol for tumors with a mean diameter $<15 \mathrm{~mm}$. Notably, conventional intraoperative residual tumor detection in CEUS has mainly depended on characteristic enhancement of tumors (35), while FI can show the spatial relationship between the original tumor and the ablation area $(36,37)$, thereby improving accuracy of evaluating intraoperative ablation edge 


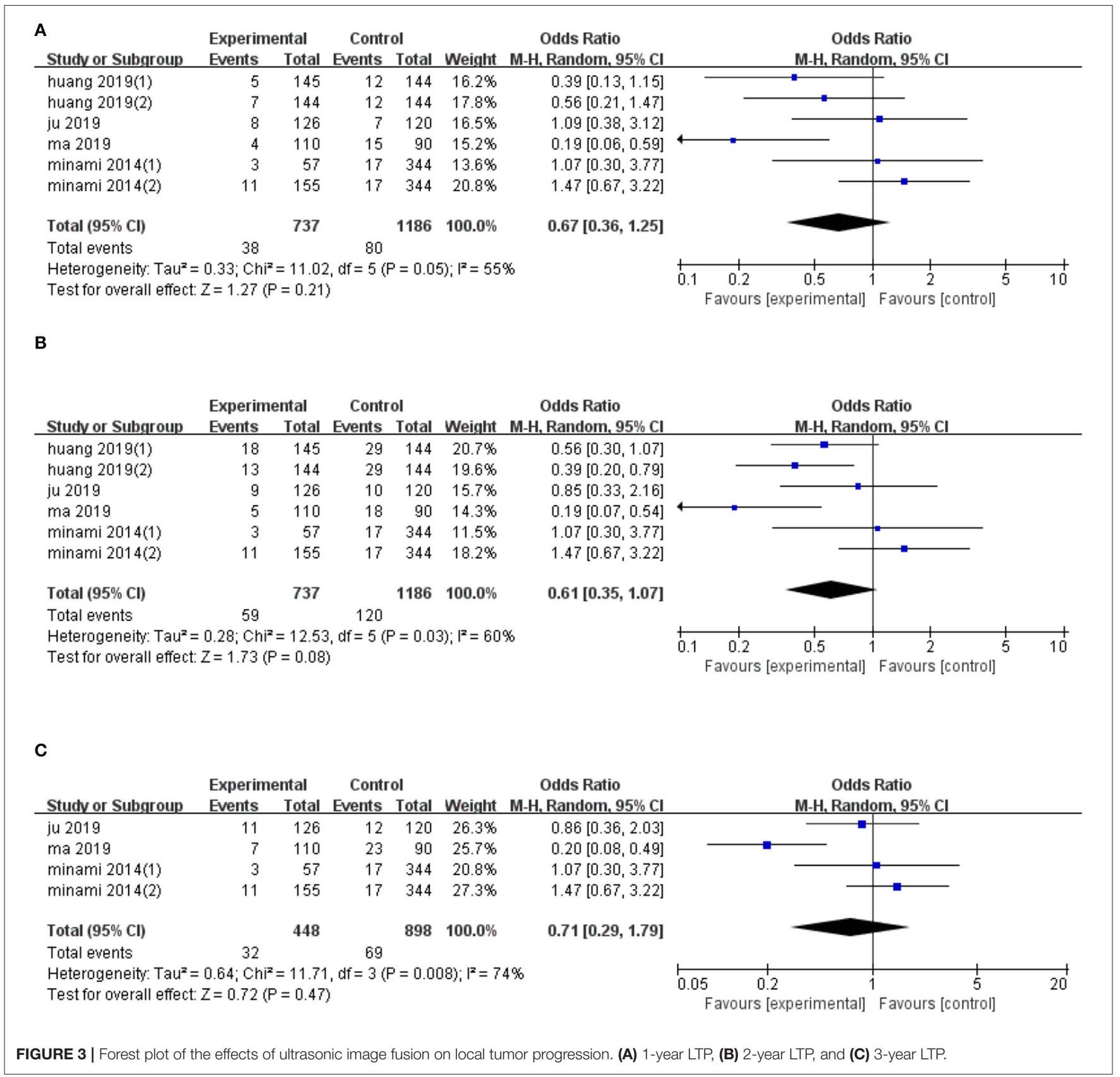

and reducing the residual tumor. Therefore, FI may improve efficacy of ablation surgery, and expand its indications $(20,21)$, which is consistent with the findings of our subgroup analyses. Specifically, this technique resulted in significantly lower 1- and 2-year LTP, relative to ultrasound in FI for tumors with a mean diameter $>15 \mathrm{~mm}$, and the subgroup analyses also confirmed that tumor diameter was the source of heterogeneity.

However, FI has its limitations. Firstly, inherent image distortion between US and CT/MR images is inevitable, especially when patients undergo changes in position, artificial ascites, pleural effusion, or other adjuvant surgery (38). Secondly, location of subcapsular tumors represents an important factor affecting misdiagnosis after fusion image-guided HCC ablation (10). Large anatomical markers, such as the portal vein branch, cannot be used for the localization of such tumors, while the rib shadow can obscure the line of sight of the tumor. These phenomenon increase the difficulty of ablation and may affect the accuracy of FI registration. It also suggests that FI may have limited effect on improving the efficacy and safety of thermal ablation of in HCC patients. So, FI was not significantly different from ultrasound in the efficiency of ablation technology, 1-3 year LTP, and 2-year OS. But the study (39) has found that the distance between the tumor and the surrounding anatomical markers $(<3 \mathrm{~cm})$ can significantly reduce the surgical efficacy using FI, 


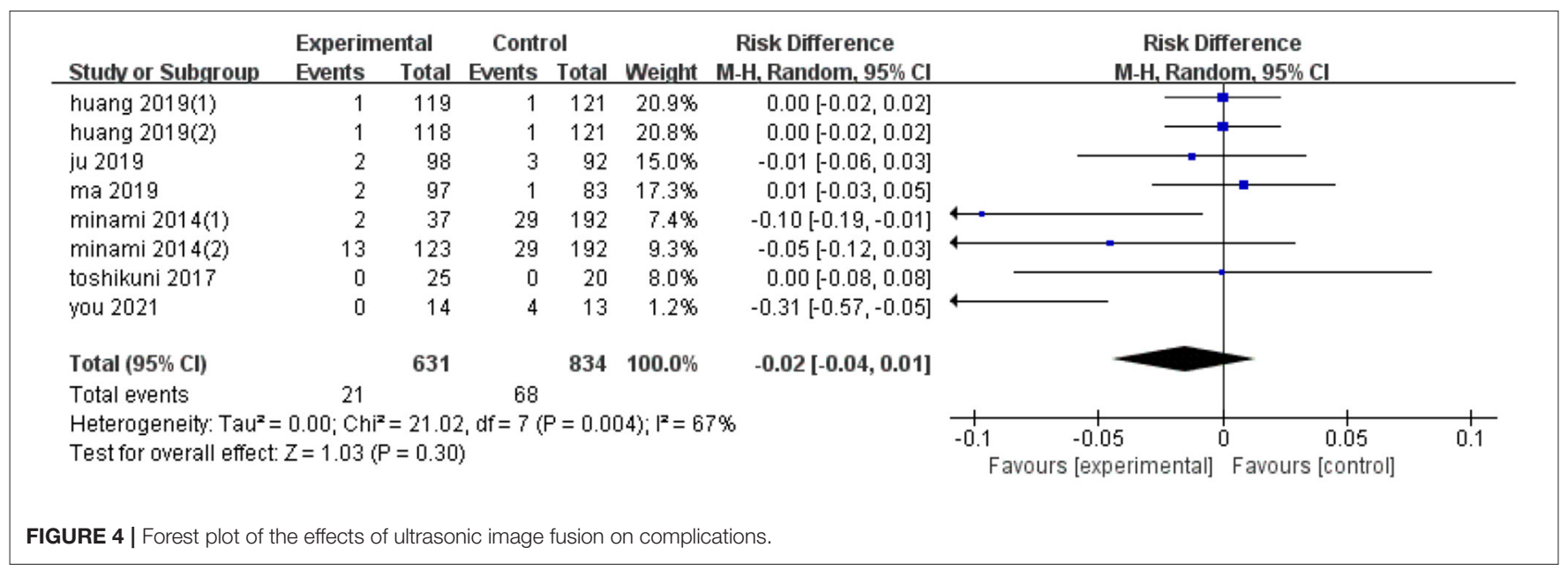

\section{A}

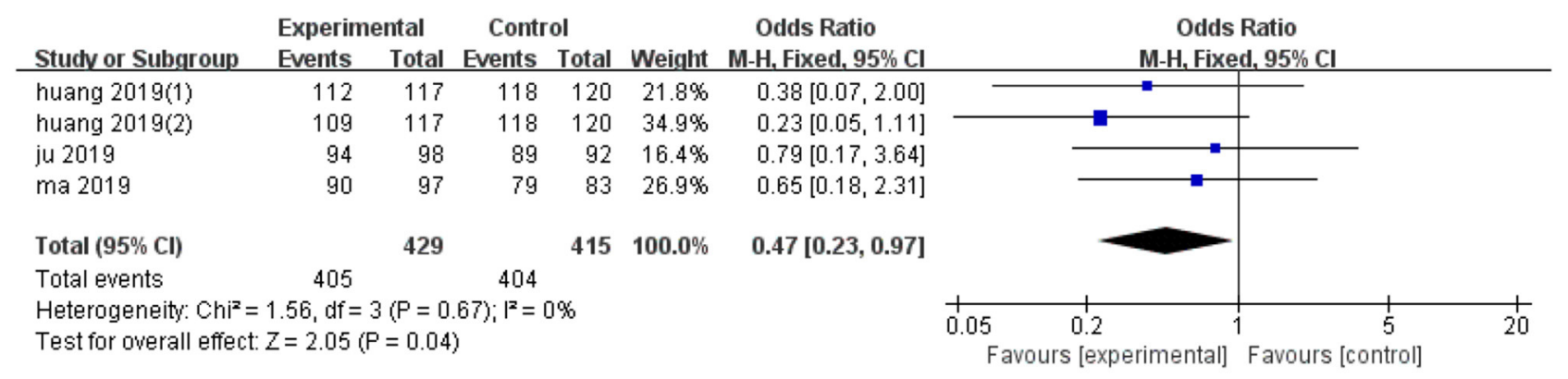

B

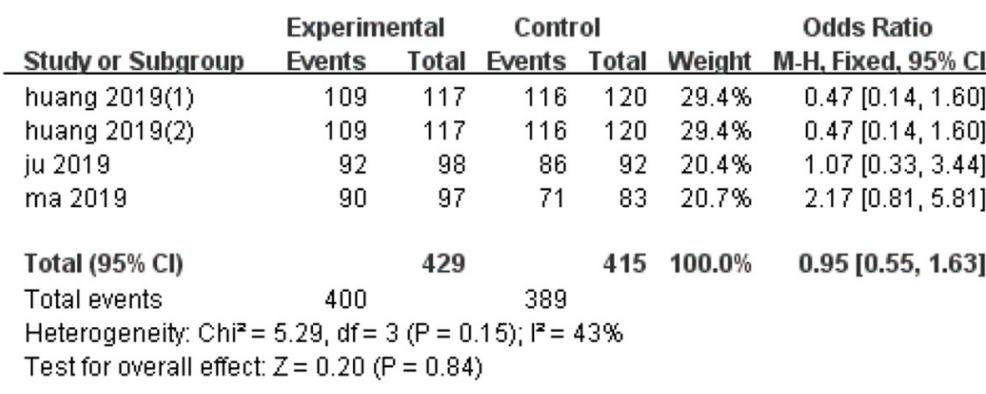

Favours [experimental] Favours [control]

FIGURE 5 | Forest plot of the effects of ultrasonic image fusion on overall survival. (A) 1-year OS and (B) 2-year OS.

which has nothing to do with the location and diameter of the tumor or the patient's voluntary breathing, etc. Therefore, further research is needed to explore the limitations of FI.

The advantage of this study is that the difference in efficacy and safety between contrastive FI and ultrasound in RFA is controversial and there has not been a relevant metaanalysis. Secondly, this study evaluated the effectiveness of the ablation technique, 1-3 years of LTP, complications, and 1-2 years of OS, and performed subgroup analysis based on tumor diameter and type of control group. In addition, any bias in the review process can be minimized by conducting a comprehensive search of the literature and by following PRISMA guidelines for conducting and reporting reviews.

The study also had some limitations. Firstly, it was difficult to conduct randomization due to the nature of the intervention, so most of the included studies are cohort studies, with a few 
high-quality studies. Secondly, differences in geographical regions, ages, and sexes of the patients might have introduced some bias (40). Thirdly, further studies are needed to investigate the indications of FI in RFA due to inconsistent definitions for evaluating liver function and difficult lesions. Fourthly, since there are many FI schemes and FI has certain efficacy for tumors of a certain diameter $(3-5 \mathrm{~cm})(41)$, it is necessary to find perfect relevant studies on the differences between FI schemes and their application value.

\section{CONCLUSION}

Currently, FI may play a role in improving the efficacy and safety of thermal ablation of HCC, compared to ultrasound, and may be more suitable for cases involving large lesions and difficult ablation. What is important is this study may provide a kind of research idea for the application value of FI. However, rigorous randomized controlled trials, with a larger sample diameter, are needed to validate these conclusions.

\section{REFERENCES}

1. Benson AB 3rd, D'Angelica MI, Abbott DE, Abrams TA, Alberts SR, Saenz DA, et al. NCCN guidelines insights: hepatobiliary cancers, Version 1, 2017. J Natl Compr Canc Netw. (2017) 15:563-73. doi: 10.6004/jnccn.2017.0059

2. Xu XL, Liu XD, Liang M, Luo BM. Radiofrequency ablation versus hepatic resection for small hepatocellular carcinoma: systematic review of randomized controlled trials with meta-analysis and trial sequential analysis. Radiology. (2018) 287:461-72. doi: 10.1148/radiol.2017162756

3. Auloge P, Cazzato RL, Koch G, Caudrelier J, De Marini P, Garnon J, et al. [Percutaneous tumor ablation]. Presse Med. (2019) 48:114655. doi: 10.1016/j.lpm.2019.10.011

4. Villanueva A. Hepatocellular carcinoma. N Engl J Med. (2019) 380:145062. doi: 10.1056/NEJMra1713263

5. Weinstein JL, Ahmed M. Percutaneous ablation for hepatocellular carcinoma. AJR Am J Roentgenol. (2018) 210:1368-75. doi: 10.2214/AJR.17.18695

6. Hui TC, Kwan J, Pua U. Advanced techniques in the percutaneous ablation of liver tumours. Diagnostics (Basel). (2021) 11:585. doi: 10.3390/diagnostics11040585

7. Izzo F, Granata V, Grassi R, Fusco R, Palaia R, Delrio P, et al. Radiofrequency ablation and microwave ablation in liver tumors: an update. Oncologist. (2019) 24:e990-e1005. doi: 10.1634/theoncologist.2018-0337

8. Xu E, Long Y, Li K, Zeng Q, Tan L, Luo L, et al. Comparison of CT/MRICEUS and US-CEUS fusion imaging techniques in the assessment of the thermal ablation of liver tumors. Int J Hyperthermia. (2019) 35:15967. doi: 10.1080/02656736.2018.1487591

9. Calandri M, Mauri G, Yevich S, Gazzera C, Basile D, Gatti M, et al. Fusion imaging and virtual navigation to guide percutaneous thermal ablation of hepatocellular carcinoma: a review of the literature. Cardiovasc Intervent Radiol. (2019) 42:639-47. doi: 10.1007/s00270-019-02167-Z

10. Ju JX, Zeng QJ, Xu EJ, He XQ, Tan L, Huang QN, et al. Intraprocedural contrast-enhanced ultrasound-CT/MR fusion imaging assessment in HCC thermal ablation to reduce local tumor progression: compared with routine contrast-enhanced ultrasound. Int J Hyperthermia. (2019) 36:78593. doi: 10.1080/02656736.2019.1640899

11. Carriero S, Della Pepa G, Monfardini L, Vitale R, Rossi D, Masperi A, et al. Role of fusion imaging in image-guided thermal ablations. Diagnostics (Basel). (2021) 11:549. doi: 10.3390/diagnostics11030549

\section{DATA AVAILABILITY STATEMENT}

The original contributions presented in the study are included in the article/supplementary material, further inquiries can be directed to the corresponding author/s.

\section{AUTHOR CONTRIBUTIONS}

TJ, SZ, and LM contributed to the research concept and design. TJ, TG, and FG participated in literature retrieval, data collection, and data analysis. TJ, FG, SZ, and LM contributed to the drafting and review of the final manuscript. All authors read and approved the final manuscript.

\section{FUNDING}

This work was supported by the National Natural Science Foundation of China (Grant No. 81601513), Young and Middleaged High-end Talents Program of Medical Research Program of Chongqing Health and Family Planning Commission (No. 2019GDRC002), and Chongqing Science and Technology Bureau (cstc2019jcyj-msxmX0837).

12. Lee DH, Lee JM. Recent advances in the image-guided tumor ablation of liver malignancies: radiofrequency ablation with multiple electrodes, real-time multimodality fusion imaging, and new energy sources. Korean J Radiol. (2018) 19:545-59. doi: 10.3348/kjr.2018.19.4.545

13. Xu E, Li K, Long Y, Luo L, Zeng Q, Tan L, et al. Intra-procedural CT/MRultrasound fusion imaging helps to improve outcomes of thermal ablation for hepatocellular carcinoma: results in 502 nodules. Ultraschall Med. (2021) 42:e9-e19. doi: 10.1055/a-1021-1616

14. Luo L, He X, Li K, Long Y, Zeng Q, Tan L, et al. Thermal ablation of medium-sized hepatocellular carcinomas using intraoperative ultrasound fusion imaging: a propensity score-matched analysis. Clin Res Hepatol Gastroenterol. (2021) 45:101581. doi: 10.1016/j.clinre.2020.11.011

15. Aj L, Kalra N, Bhatia A, Srinivasan R, Gulati A, Kapoor R, et al. Fusion image-guided and ultrasound-guided fine needle aspiration in patients with suspected hepatic metastases. J Clin Exp Hepatol. (2019) 9:54753. doi: 10.1016/j.jceh.2019.01.003

16. Boesen L. Magnetic resonance imaging-transrectal ultrasound image fusion guidance of prostate biopsies: current status, challenges and future perspectives. Scand J Urol. (2019) 53:8996. doi: 10.1080/21681805.2019.1600581

17. Kobe A, Kindler Y, Klotz E, Puippe G, Messmer F, Alkadhi H, et al. Fusion of preinterventional MR imaging with liver perfusion CT after RFA of hepatocellular carcinoma: early quantitative prediction of local recurrence. Invest Radiol. (2021) 56:188-96. doi: 10.1097/RLI.0000000000000726

18. Nobre C, Oliveira-Santos M, Paiva L, Costa M, Gonçalves L. Fusion imaging in interventional cardiology. Rev Port Cardiol. (2020) 39:46373. doi: $10.1016 /$ j.repc.2020.03.014

19. Wu DF, He W, Lin S, Zee CS, Han B. The real-time ultrasonography for fusion image in glioma neurosugery. Clin Neurol Neurosurg. (2018) 175:8490. doi: 10.1016/j.clineuro.2018.10.009

20. Long Y, Xu E, Zeng Q, Ju J, Huang Q, Liang $\mathrm{P}$, et al. Intra-procedural real-time ultrasound fusion imaging improves the therapeutic effect and safety of liver tumor ablation in difficult cases. Am J Cancer Res. (2020) 10:2174-84.

21. Ma QP, Xu EJ, Zeng QJ, Su ZZ, Tan L, Chen JX, et al. Intraprocedural computed tomography/magnetic resonance-contrast-enhanced ultrasound fusion imaging improved thermal ablation effect of hepatocellular carcinoma: comparison with conventional ultrasound. Hepatol Res. (2019) 49:799809. doi: $10.1111 /$ hepr.13336 
22. Huang Q, Zeng Q, Long Y, Tan L, Zheng R, Xu E, et al. Fusion imaging techniques and contrast-enhanced ultrasound for thermal ablation of hepatocellular carcinoma - a prospective randomized controlled trial. Int J Hyperthermia. (2019) 36:1207-15. doi: 10.1080/02656736.2019.1687945

23. You Y, Long Y, Yan R, Luo L, Zhang M, Li L, et al. Improving ablation safety for hepatocellular carcinoma proximal to the hilar bile ducts by ultrasoundMR fusion imaging: a preliminary comparative study. Front Oncol. (2021) 11:570312. doi: 10.3389/fonc.2021.570312

24. Shiina S, Sato K, Tateishi R, Shimizu M, Ohama H, Hatanaka T, et al. Percutaneous ablation for hepatocellular carcinoma: comparison of various ablation techniques and surgery. Can J Gastroenterol Hepatol. (2018) 2018:4756147. doi: 10.1155/2018/4756147

25. Toshikuni N, Matsue Y, Ozaki K, Yamada K, Hayashi N, Tsuchishima M, et al. An image fusion system for estimating the therapeutic effects of radiofrequency ablation on hepatocellular carcinoma. Radiol Oncol. (2017) 51:263-9. doi: 10.1515/raon-2017-0028

26. Minami T, Minami Y, Chishina H, Arizumi T, Takita M, Kitai S, et al. Combination guidance of contrast-enhanced US and fusion imaging in radiofrequency ablation for hepatocellular carcinoma with poor conspicuity on contrast-enhanced US/fusion imaging. Oncology. (2014) 87(Suppl. 1) 5562. doi: $10.1159 / 000368146$

27. Bray F, Ferlay J, Soerjomataram I, Siegel RL, Torre LA, Jemal A. Global cancer statistics 2018: GLOBOCAN estimates of incidence and mortality worldwide for 36 cancers in 185 countries. CA Cancer J Clin. (2018) 68:394424. doi: $10.3322 /$ caac. 21492

28. Zhu F, Rhim H. Thermal ablation for hepatocellular carcinoma: what's new in 2019. Chin Clin Oncol. (2019) 8:58. doi: 10.21037/cco.2019.11.03

29. Wang F, Numata K, Nihonmatsu H, Okada M, Maeda S. Application of new ultrasound techniques for focal liver lesions. J Med Ultrason. (2020) 47:215-37. doi: 10.1007/s10396-019-01001-w

30. Thamtorawat S, Hicks RM, Yu J, Siripongsakun S, Lin WC, Raman SS, et al. Preliminary outcome of microwave ablation of hepatocellular carcinoma: breaking the 3-cm barrier? J Vasc Interv Radiol. (2016) 27:62330. doi: 10.1016/j.jvir.2016.01.011

31. Ahn SJ, Lee JM, Lee DH, Lee SM, Yoon JH, Kim YJ, et al. Real-time US-CT/MR fusion imaging for percutaneous radiofrequency ablation of hepatocellular carcinoma. J Hepatol. (2017) 66:347-54. doi: 10.1016/j.jhep.2016. 09.003

32. Hakime A, Yevich S, Tselikas L, Deschamps F, Petrover D, De Baere T. Percutaneous thermal ablation with ultrasound guidance, fusion imaging guidance to improve conspicuity of liver metastasis. Cardiovasc Intervent Radiol. (2017) 40:721-7. doi: 10.1007/s00270-016-1561-5

33. Schwarze V, Rübenthaler J, Marschner C, Fabritius MP, Rueckel J, Fink N, et al. Advanced fusion imaging and contrast-enhanced imaging (CT/MRICEUS) in oncology. Cancers (Basel). (2020) 12:2821. doi: 10.3390/cancers121 02821

34. Song KD, Lee MW, Rhim H, Kang TW, Cha DI, Sinn DH, et al. Percutaneous US/MRI fusion-guided radiofrequency ablation for recurrent subcentimeter hepatocellular carcinoma: technical feasibility and therapeutic outcomes. Radiology. (2018) 288:878-86. doi: 10.1148/radiol.2018 172743

35. Xuan M, Zhou F, Ding Y, Zhu Q, Dong J, Zhou H, et al. Diagnostic accuracy of contrast-enhanced ultrasound in assessing the therapeutic response to radio frequency ablation for liver tumors: systematic review and meta-analysis. Surg Endosc. (2018) 32:2067-75. doi: 10.1007/s00464-017-5903-4

36. Ye J, Huang G, Zhang X, Xu M, Zhou X, Lin M, et al. Threedimensional contrast-enhanced ultrasound fusion imaging predicts local tumor progression by evaluating ablative margin of radiofrequency ablation for hepatocellular carcinoma: a preliminary report. Int J Hyperthermia. (2019) 36:55-64. doi: 10.1080/02656736.2018.1530460

37. Li K, Su Z, Xu E, Huang Q, Zeng Q, Zheng E. Evaluation of the ablation margin of hepatocellular carcinoma using CEUS-CT/MR image fusion in a phantom model and in patients. BMC Cancer. (2017) 17:61. doi: 10.1186/s12885-017-3061-7

38. Minami Y, Kudo M. Image guidance in ablation for hepatocellular carcinoma: contrast-enhanced ultrasound and fusion imaging. Front Oncol. (2021) 11:593636. doi: 10.3389/fonc. 2021.593636

39. Calandri M, Ruggeri V, Carucci P, Mirabella S, Veltri A, Fonio P, et al. Thermal ablation with fusion imaging guidance of hepatocellular carcinoma without conspicuity on conventional or contrast-enhanced US: surrounding anatomical landmarks matter. Radiol Med. (2019) 124:10438. doi: 10.1007/s11547-019-01057-1

40. Tang A, Hallouch O, Chernyak V, Kamaya A, Sirlin CB. Epidemiology of hepatocellular carcinoma: target population for surveillance and diagnosis. Abdom Radiol (NY). (2018) 43:13-25. doi: 10.1007/s00261-017-1209-1

41. Luo L, Yan R, Li K, Zeng Q, Tan L, Long Y, et al. The value of ultrasound fusion imaging technology in assisting thermal ablation of $35 \mathrm{~cm}$ liver cancer. Chin J Ultrasound Imaging. (2019) 28:1102-1103. doi: 10.3760/cma.j.issn.1004-4477.2019.04.008

Conflict of Interest: The authors declare that the research was conducted in the absence of any commercial or financial relationships that could be construed as a potential conflict of interest.

Publisher's Note: All claims expressed in this article are solely those of the authors and do not necessarily represent those of their affiliated organizations, or those of the publisher, the editors and the reviewers. Any product that may be evaluated in this article, or claim that may be made by its manufacturer, is not guaranteed or endorsed by the publisher.

Copyright (c) 2021 Jie, Guoying, Gang, Zhengrong and Maoping. This is an openaccess article distributed under the terms of the Creative Commons Attribution License (CC BY). The use, distribution or reproduction in other forums is permitted, provided the original author(s) and the copyright owner(s) are credited and that the original publication in this journal is cited, in accordance with accepted academic practice. No use, distribution or reproduction is permitted which does not comply with these terms. 